\title{
Transfer of Negative Muons to Gases Dissolved in a Hydrogen Bubble Chamber.
}

\author{
M. SCHIFF \\ The Enrico Fermi Institute for Nuclear Studies
}

The Department of Physics, The University of Chicago - Chicago, Ill.

(Nuovo Cimento, 22, $66(1961))$

On the top right of Fig. 6 on p. 80

instead of $1600 \mathrm{Ne} \quad$ read $1600 \mathrm{He}$. 